\title{
REVISIÓN SISTEMÁTICA: VALORACIÓN AMBIENTAL EN LA AGRICULTURA
}

\section{SYSTEMATIC REVIEW: ENVIRONMENTAL ASSESSMENT IN AGRICULTURE}

\author{
Antonio Ismael Acevedo Peralta* \\ Juan Antonio Leos Rodríguez ${ }^{* *}$ \\ Uriel Figueroa Viramontes* \\ José Luis Romo Lozano****
}

RESUMEN

El artículo analiza 24 investigaciones realizadas en diferentes países entre los años 2008 al 2015, sobre cuestiones ambientales generadas por la agricultura, así como, se discute la validez de los métodos de valoración económica utilizados. Los métodos incluidos son: valoración contingente, experimentos de elección, precios hedónicos y valoración de activos multicriterio. La mayoría de las investigaciones se han centrado en la recreación y el turismo, dejando de lado los problemas ocasionados por la agricultura. Al final, se ofrecen algunas recomendaciones relacionadas con la valoración económica del ambiente en el diseño de políticas enfocadas al sector agrícola.

PALABRAS CLAVE: AMBIENTE * AGRICULTURA * EVALUACIÓN ECONÓMICA * POLÍTICA PÚBLICA

\section{ABSTRACT}

The analysis included 24 studies, conducted in different countries, on environmental issues generated from agriculture. Also, the validity of the methods is discussed. The methods included are: contingent valuation, choice experiments, hedonic prices and multicriteria valuation assets. Most research has focused on recreation and tourism, leaving aside the

\footnotetext{
* Centro de Investigaciones Económicas, Sociales y Tecnológicas de la Agroindustria y Agricultura Mundial (CIESTAAM), Universidad Autónoma Chapingo, México. iacevedo@ciestaam.edu.mx

** Centro de Investigaciones Económicas, Sociales y Tecnológicas de la Agroindustria y Agricultura Mundial (CIESTAAM), Universidad Autónoma Chapingo, México. jleos@ciestaam.edu.mx

*** Instituto Nacional de Investigaciones Forestales, Agrícolas y Pecuarias (INIFAP), Campo Experimental La Laguna, México.

figueroa.uriel@inifap.gob.mx

**** División de Ciencias Forestales (DICIFO), Universidad Autónoma Chapingo, México. lromo@aya.yale.edu
} 
problems caused by agriculture. In the end, some recommendations related to economic valuation of the environment in the design of policies focused on the agricultural sector.

KEYWORDS: ENVIRONMENT * AGRICULTURE * ECONOMIC EVALUATION * GOVERNMENT POLICY

\section{INTRODUCCIÓN}

El gobierno tiene una obligada injerencia en el manejo y gestión del ambiente, proceso que requiere acciones colectivas (normativas), la coordinación pública y privada, $y$ la participación social en la toma de decisiones. Así, las políticas adecuadas pueden no solo mantener las condiciones ambientales, sino mejorarlas simultáneamente junto al desarrollo económico; por lo que es necesario contemplar dos tipos de políticas (World Bank 1992): las que construyen relaciones positivas entre desarrollo $y$ ambiente, $y$ las que reducen o eliminan las relaciones negativas.

La agricultura puede ser objeto de contaminación al ser el receptor de la misma por medio del ambiente; así como puede ser sujeto al contribuir de forma importante en externalidades negativas debido a la cantidad de insumos que se utilizan en la producción. Para el caso de México, las externalidades se manifestan en problemas de salinización, acidificación, erosión, compactación y desertificación del suelo, así como erosión genética la cual afecta cerca del $80 \%$ del territorio nacional. Hay pérdida de biodiversidad y deforestación en cerca de 500 a 700 mil hectáreas por año. Además de contaminación de suelos, agua y atmósfera, sedimentación, efecto invernadero y cambio climático, debido a la liberación de entre 1.6 y 2.4 Pg de carbono por año (Pérez y Landeros 2009).

Una de las alternativas para la solución de la problemática anterior consiste en estimar el valor monetario de aquellos bienes $y$ servicios ambientales, así como, las externalidades que carecen de mercado. En la actualidad, los métodos de valoración económica son una herramienta que permiten asignar valor a los servicios ambientales, sobre todo en el sistema económico actual en donde las decisiones se basan en valores monetarios, para reintegrar los efectos negativos en la toma de decisiones (Haro y Taddei 2010).
Por lo tanto, es importante identificar los principales métodos utilizados; una herramienta de fácil manejo es la revisión sistemática, que implica identificar toda la evidencia existente desarrollada por las comunidades académicas, evaluar críticamente su calidad y generar una síntesis capaz de formular recomendaciones que se deriven de estas (Torgerson 2003 y Flores 2013). Así, las revisiones sistemáticas permiten identificar lo que falta por saber, acceder a evidencia rigurosa e incorporar la evidencia existente en el proceso de diseño de políticas públicas de manera explícita y replicable (Sanz 2013).

Debido a las características interdisciplinarias de los problemas ambientales y el rol que juega la agricultura, el presente documento hace un acercamiento al tema a partir del objetivo de investigación orientado a analizar las diferentes metodologías de valoración económica que se han utilizado en la agricultura, mediante una revisión sistemática, para identificar los factores que más influyen en la disposición a pagar y extraer resultados que se puedan incorporar como recomendaciones en el diseño de políticas públicas que ayuden a mitigar los impactos en el medio ambiente.

\section{MATERIALES Y MÉTODOS}

\section{OBTENCIÓN DE LA INFORMACIÓN}

La búsqueda de los artículos se realizó en diferentes bases de datos, las cuales fueron EBSCOhost, ELSEVIER, SPRINGER, JSTOR, ScIELO, Redalyc, Taylor \& Francis, Cambridge University Press y Oxford Journals. La búsqueda se realizó tanto en inglés como en español y no se introdujo ninguna restricción en los años de búsqueda, ni en el tipo de documento, para obtener el mayor número de trabajos posibles. Los términos de búsqueda se limitaron al título, resumen y palabras clave, relacionadas con los métodos de valoración económica de los impactos de la agricultura. 


\section{CRITERIOS DE INCLUSIÓN Y EXCLUSIÓN}

Se incluyeron solo trabajos en los que se emplearon métodos de valoración económica de forma específica y claramente operacionalizados en el sector agrícola. Este criterio permitió descartar todos aquellos trabajos que incluían valoración económica de manera general o enfocada a otros sectores no agrícolas, y aquellos en los que la operacionalización no dejaba en claro si se trataba de cuestiones ambientales relacionadas con las actividades agrícolas o con otras actividades. Se tomaron en consideración trabajos que reportaron datos empíricos originales y recientes, realizados del año 2008 al año 2015. Se excluyeron informes de caso, revisiones de evaluaciones económicas, opiniones de expertos publicadas como editoriales o cartas al editor, artículos metodológicos y trabajos no publicados en revistas con factor de impacto, este criterio garantiza que los documentos se encuentren disponibles en la comunidad académica de diversos países.

\section{CODIFICACIÓN DE LOS RESULTADOS}

Los estudios se sintetizaron en tablas prediseñadas y se extrajo la siguiente información: autor/es y año de publicación; lugar donde se realizó el estudio; objeto de estudio de la investigación, relacionado con el ambiente $y$ derivado de las actividades agrícolas; muestra/población descritos en el estudio; método de valoración económica empleado e impacto de los resultados.

\section{VALIDACIÓN DE LOS MÉTODOS}

Se realizó una evaluación de la metodología empleada (cuadro 1), donde se especifican las variables de análisis por cada método, esto con la finalidad de juzgar la validez interna $y$ externa. La validez interna se define como la capacidad de un estudio para establecer la causalidad; mientras que la validez externa como la capacidad de un estudio para establecer las relaciones causales que pueden ser extrapolables a zonas o países distintos de aquellos en que se llevó a cabo originalmente el estudio.

CUADRO 1

VARIABLES ANALIZADAS EN LOS MÉTODOS DE VALORACIÓN ECONÓMICA

\begin{tabular}{|c|c|c|c|}
\hline \multirow{2}{*}{ MÉTODO } & \multicolumn{3}{|c|}{ VARIABLES } \\
\hline & ESTUDIOS & DISPOSICIÓN A PAGAR & PROBLEMÁTICA \\
\hline $\begin{array}{l}\text { Valoración } \\
\text { contingente }\end{array}$ & 10 & $\begin{array}{l}\text { Factores socioeconómicos } \\
\text { Actividades agrícolas } \\
\text { Regulación de servicios } \\
\text { Disponibilidad de agua } \\
\text { Programas de conservación } \\
\text { Manejo de desechos }\end{array}$ & $\begin{array}{l}\text { Residuos y desechos agrícolas } \\
\text { Ecosistemas sostenibles } \\
\text { Agua de riego } \\
\text { Multifuncionalidad de la agricultura } \\
\text { Recursos genéticos locales }\end{array}$ \\
\hline $\begin{array}{l}\text { Experimentos de } \\
\text { elección }\end{array}$ & 4 & $\begin{array}{l}\text { Disponibilidad de agua } \\
\text { Precio del agua } \\
\text { Parcelas de producción } \\
\text { Calidad ambiental }\end{array}$ & $\begin{array}{l}\text { Agua para riego } \\
\text { Zonas de amortiguación } \\
\text { Paisajes agrícolas } \\
\text { Contaminación }\end{array}$ \\
\hline Precios hedónicos & 2 & $\begin{array}{l}\text { Calidad del aire } \\
\text { Disminución de ruido } \\
\text { Servicios recreativos }\end{array}$ & $\begin{array}{l}\text { Malos olores } \\
\text { Transporte de estiércol } \\
\text { Paisajes agrícolas }\end{array}$ \\
\hline MVAM (PJA+PM) & 1 & $\begin{array}{l}\text { Valoración de activos de producción } \\
\text { Manejo del suelo }\end{array}$ & $\begin{array}{l}\text { Insumos de producción } \\
\text { Prácticas de producción }\end{array}$ \\
\hline $\begin{array}{l}\text { Combinación de } \\
\text { métodos }\end{array}$ & 7 & $\begin{array}{l}\text { Impuestos } \\
\text { Componentes de producción } \\
\text { Atributos de paisaje } \\
\text { Preservación de tierras agrícolas }\end{array}$ & $\begin{array}{l}\text { Gases de efecto invernadero } \\
\text { Paisajes agrícolas }\end{array}$ \\
\hline
\end{tabular}

Fuente: Elaboración propia. 


\section{RESULTADOS Y DISCUSIÓN}

El procedimiento de búsqueda dio lugar a 116 documentos potencialmente relevantes, sin embargo, una vez que se aplicaron los criterios de inclusión y exclusión, se consideraron dentro de esta revisión sistemática 24. En la figura 1 se presenta el flujograma del proceso de selección de estos estudios.

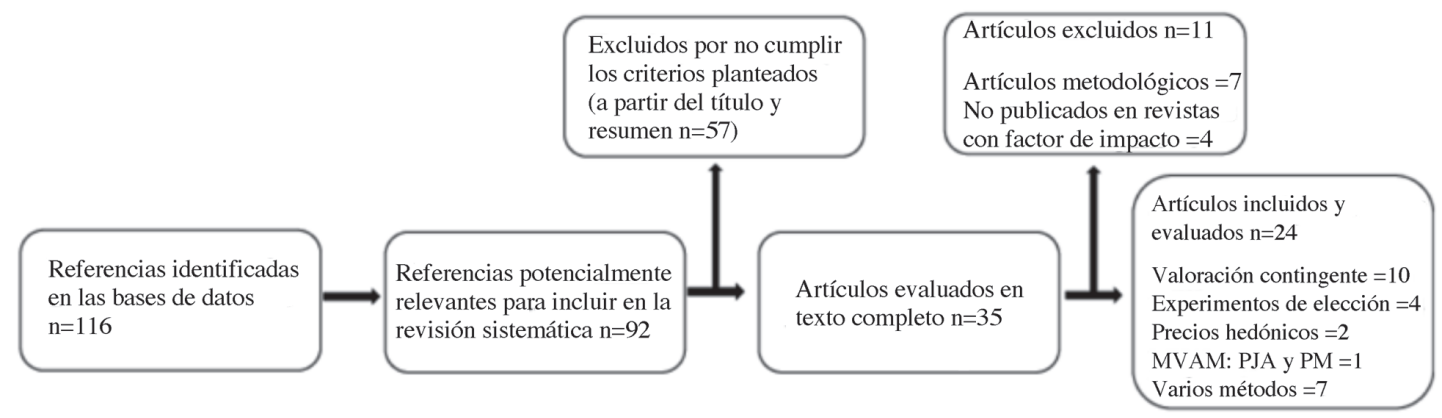

FIGURA 1

FLUJOGRAMA DEL PROCESO DE SELECCIÓN DE ESTUDIOS

Fuente: Elaboración propia.

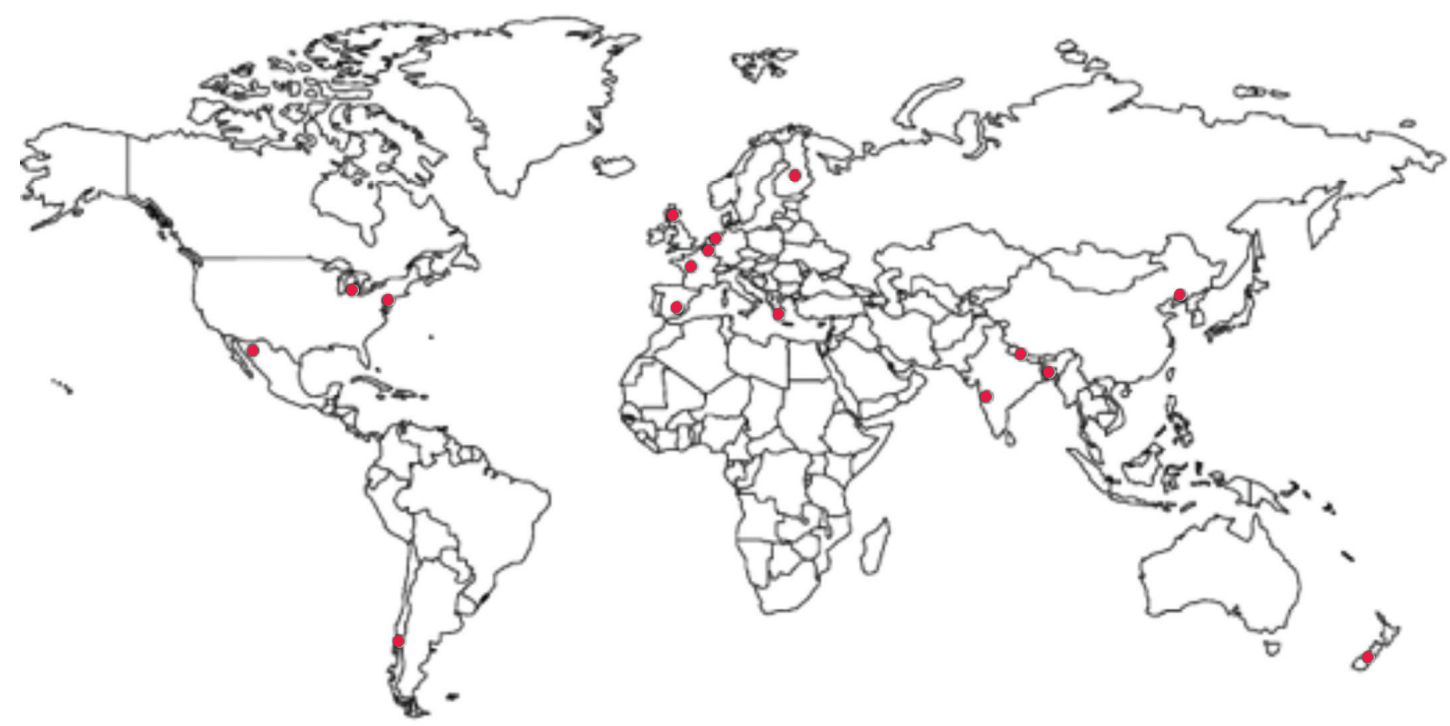

FIGURA 2

UBICACIÓN DE LOS ESTUDIOS DE VALORACIÓN

Fuente: Elaboración propia. 
Los trabajos se agruparon con base en el método de valoración empleado. El 41,67\% de los estudios corresponde al uso del método de valoración contingente, el 16,67\% a experimentos de elección, el 8,33\% utiliza el método de precios hedónicos, el 4,17\% utiliza el método de valoración de activos multicriterio (MVAM), integrado por el proceso jerárquico analítico (PJA) y la programación por metas (PM); y el $29,17 \%$ restante de los estudios utiliza una combinación de los métodos anteriores, en el que prevalece la valoración contingente. En los últimos cuatro años, del 2011 al 2014, se concentra el 75\% de las publicaciones utilizadas en esta investigación y el 25\% restante se publicó antes de 2011. La figura 2 muestra los diferentes países donde se realizaron los estudios de valoración económica.

\section{VALIDACIÓN DE LOS MÉTODOS EVALUADOS}

Ryan (2011) menciona que el carácter normativo de los servicios culturales y la heterogeneidad en la valoración de los actores de la sociedad han hecho su cuantificación más difícil. Aun así, un número de estudios se han desarrollado para la disposición de los servicios valorados por las partes interesadas, donde se incluyen los servicios ambientales, a través de las actividades agrícolas.

\section{VALORACIÓN CONTINGENTE}

El método que predomina en los trabajos analizados es la valoración contingente, donde el valor económico se obtiene de forma directa de las respuestas de los entrevistados. Los principales resultados que derivaron de la aplicación de este método se ven influenciados por las variables socioeconómicas consideradas en las encuestas aplicadas, sobresaliendo el nivel de ingresos y la educación del entrevistado, lo que permite un mayor conocimiento del problema e influye positivamente en la disposición a pagar (DAP), tal y como lo establece la teoría económica (cuadro 2). En este sentido, Kristrom (1995) señala que cuando hay una relación positiva entre la variable ingreso y la disposición a pagar, los bienes ambientales son bienes normales.

Contrariamente, en un estudio realizado por Riera (1994) se reporta que no encontró una relación positiva entre los ingresos más altos y la DAP, incluso se apreció una relación inversa, es decir, personas con ingresos más bajos presentaron una mayor DAP, con lo que el autor concluyó que los bienes naturales no son bienes de lujo. Por tal motivo, se puede inferir que no necesariamente las personas que poseen mayores ingresos $y$ un mayor nivel de educación están dispuestas a pagar más.

Las personas con un menor ingreso presentan una menor probabilidad a pagar, pues esto conlleva a un costo lo que es consistente con las teorías del consumidor (Lancaster 1966). Es importante resaltar que la mayoría de las personas estarían dispuestas a pagar por un bien o servicio ambiental, pues reconocen en su atributo un beneficio para mejorar su bienestar, sin embargo, la brecha para el acceso se encuentra en los recursos económicos de los cuales disponen. 


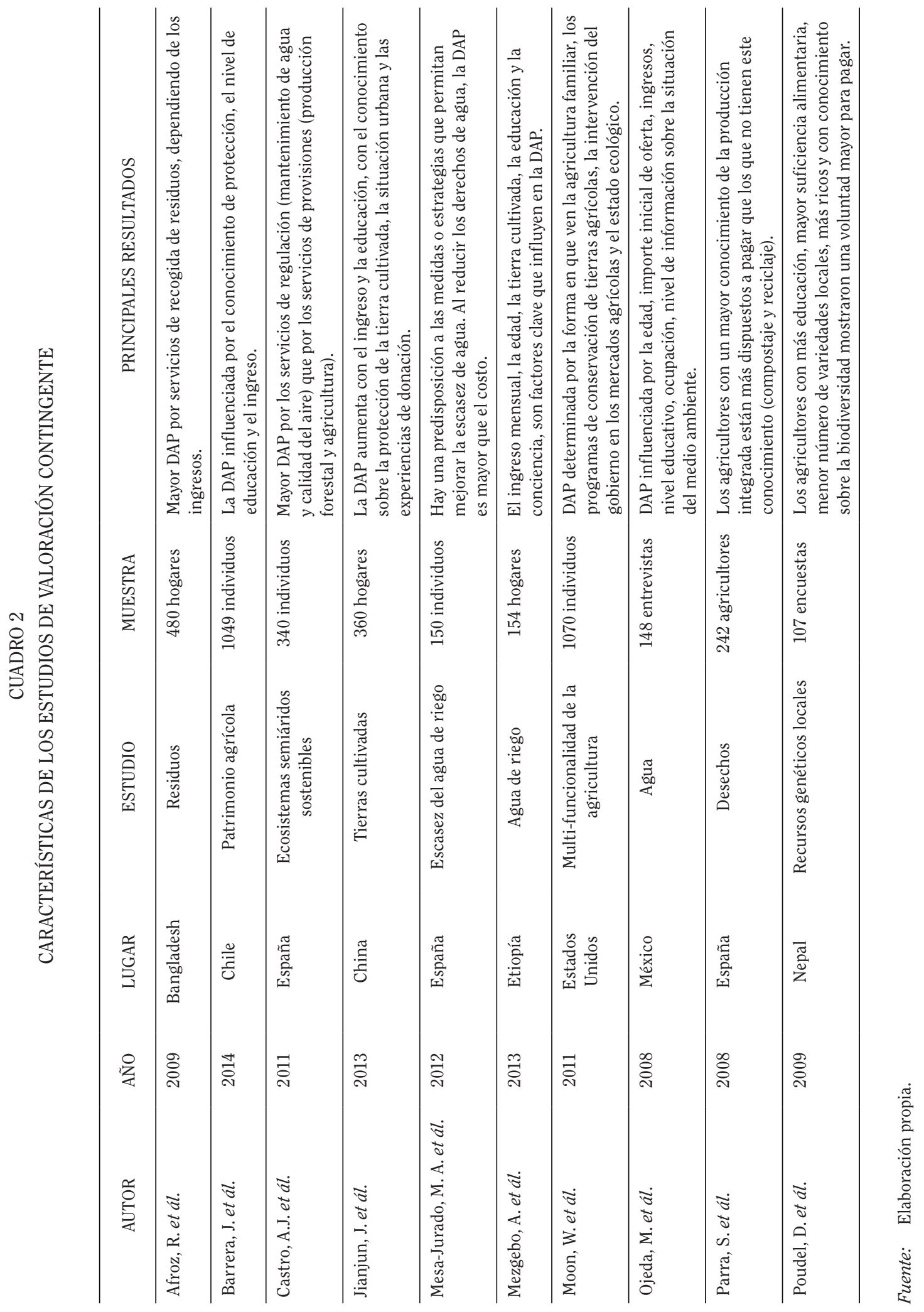

(c) (1) $€$ Rev. Ciencias Sociales Universidad de Costa Rica, 152: 89-105 / 2016 (II). (ISSN: 0482-5276) 
Sin embargo, diferentes autores revelan que la mayor crítica de los resultados obtenidos de la aplicación de este método gira principalmente alrededor de dos aspectos: la validez y la fiabilidad (Smith 1993, Freeman 2003 y NOAA 1993). En términos simples, para Kealy et ál. (1990), la validez se refiere a la exactitud y la fiabilidad se refiere a la coherencia o reproducibilidad de los resultados.

Para Hausman (2012) existen algunos problemas en los estudios de valoración contingente en situaciones reales de mercado, donde se puede afectar las opciones del consumidor debido a que tienen una restricción presupuestaria, con efecto en sus decisiones. Así, este método es inaceptable pues refleja los valores de existencia y para que sean aceptados en un análisis de costo-beneficio, sería necesario que los valores de existencia de cada persona reflejen únicamente sus motivos económicos individuales y no sus motivos altruistas, de sentido del deber o de obligación moral (Diamond et ál.1993).

Carson y Groves (2007) argumentan que los resultados se deben a la naturaleza de los bienes privados. Debido a que los encuestados no están obligados a comprar el bien, podrían actuar de forma estratégica al exagerar su DAP si creen que esto alentaría un nuevo bien para estar disponible en el mercado. Sin embargo, los bienes públicos se proporcionan en un contexto diferente. El gobierno puede proveer bienes públicos y obligar a todos a pagar, a través de los impuestos. Si los participantes de la encuesta creen que pueden ser obligados a pagar basados en sus respuestas, puede que ya no tengan un incentivo para responder de manera estratégica.

A pesar de las críticas del método, cabe señalar que la valoración contingente se ha convertido en una parte integral de evaluación ambiental. Sin embargo, Venkatachalam (2004) menciona que algunos estudios se han llevado a cabo de forma indiscriminada, sin evaluar la validez y fiabilidad de los resultados siendo menos relevantes para el propósito de formulación de políticas. Se recomienda que los estudios donde se utilice este método deben ser diseñados de tal manera que las "pruebas de coherencia interna" (Smith y Osborne 1996) puedan llevarse a cabo para evaluar la validez y la fiabilidad de los resultados.

\section{EXPERIMENTOS DE ELECCIÓN}

Los experimentos de elección son esencialmente un procedimiento diseñado para revelar los factores que influyen en las preferencias individuales, reúnen la noción de atributos y la noción de impacto ambiental evaluados; por lo que los usuarios de este método pueden centrarse en el aspecto multidimensional de la intervención pública y al mismo tiempo dar valor económico a los impactos de los diferentes componentes de la política, $y$ al impacto global de un paquete de políticas. Los entrevistados siempre ponen de manifiesto las altas preferencias por los atributos del ecosistema que le proporcionen bienestar (cuadro 3). 


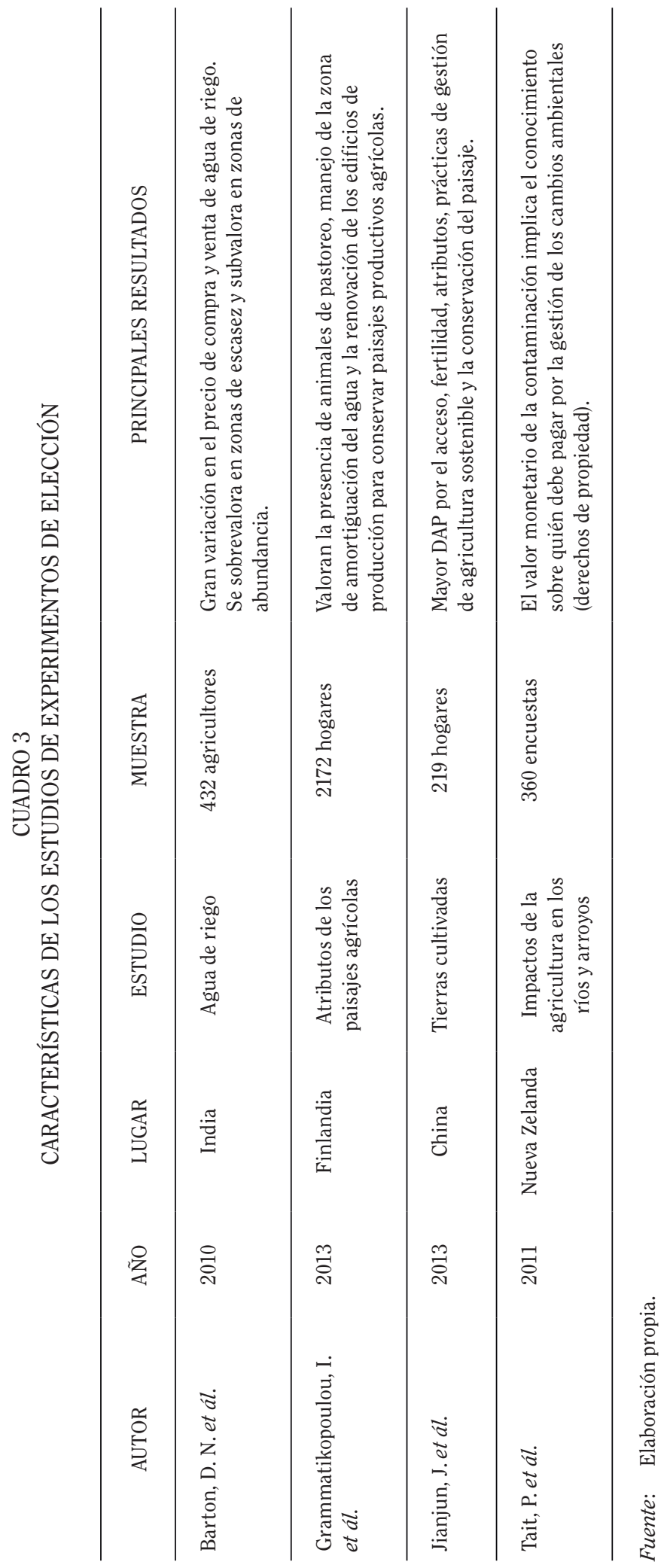

CC) (1) $\fallingdotseq$ Rev. Ciencias Sociales Universidad de Costa Rica, 152: 89-105 / 2016 (II). (ISSN: 0482-5276) 
Se ha dado un amplio debate sobre la posibilidad de inducir preferencias por los productos no comerciales en las encuestas hipotéticas (Diamond y Hausman 1994, Hanemann 1994). Asumen la posición de que no deben medirse los valores de no uso, que están motivados en gran parte por la "satisfacción moral de compra” (Kahneman y Knetsch 1992), siendo mucho más baja en una encuesta en situación hipotética que en una situación real; aunque los métodos de preferencias declaradas son esencialmente los únicos métodos disponibles para medir valores de no uso.

Sin embargo, para Alpízar et ál. (2001), los experimentos de elección pueden ser menos propensos a desencadenar este tipo de comportamiento que las encuestas de valoración contingente. La razón de esto es que en un experimento de elección individual se tienen que hacer intercambios entre varios atributos, los cuales pueden contener valores de no uso.

En este sentido, en la investigación realizada por Louviere (1988), se concluye que los experimentos de elección constituyen un instrumento válido para explicar y predecir el comportamiento individual en los mercados reales. Además, Carlsson y Martinson (2001) observaron que la DAP medida para un bien público no difiere mucho entre un experimento de elección con pagos hipotéticos y un experimento de elección con pagos reales.

\section{PRECIOS HEDÓNICOS}

El método de los precios hedónicos toma la utilidad generada de las características subyacentes de los bienes y servicios (cuadro 4), exhibe las elecciones individuales en el equilibrio del mercado (Lancaster 1966 y Rosen 1974). Ma y Swinton (2011), sugieren que se capitalizan en gran medida los valores de uso directo, como los servicios recreativos y estéticos. Además de algunos servicios de regulación que proporcionan un valor de uso indirecto (agua, suelo y clima) pueden ser parcialmente capitalizados. Se puede decir entonces que la capitalización de los servicios que proporcionan los ecosistemas de los paisajes rurales es un efecto neto que depende del equilibrio entre los diferentes servicios.

CUADRO 4

CARACTERÍSTICAS DE LOS ESTUDIOS DE PRECIOS HEDÓNICOS

\begin{tabular}{|c|c|c|c|c|c|}
\hline AUTOR & AÑO & LUGAR & ESTUDIO & MUESTRA & PRINCIPALES RESULTADOS \\
\hline $\begin{array}{l}\text { Eyckmans, } \\
\text { J.et ál. }\end{array}$ & 2013 & Bélgica & Malos olores & $\begin{array}{l}1420 \\
\text { registros }\end{array}$ & $\begin{array}{l}\text { Los camiones cargados con residuos ani- } \\
\text { males causan considerables costos externos } \\
\text { (calidad del aire y ruido) para los habitantes } \\
\text { locales, lo cual afecta el precio de las casas; } \\
\text { el costo externo total es aproximadamente } \\
31 \text { millones de euros, en el valor de las vivi- } \\
\text { endas capitalizadas. }\end{array}$ \\
\hline
\end{tabular}

\begin{tabular}{|c|c|c|c|c|c|}
\hline Ma, S. et ál. & 2011 & $\begin{array}{l}\text { Estados } \\
\text { Unidos }\end{array}$ & $\begin{array}{l}\text { Paisajes } \\
\text { agrícolas }\end{array}$ & 220 parcelas & $\begin{array}{l}\text { Los ecosistemas se capitalizan en gran } \\
\text { medida a través de los lagos, ríos, humeda- } \\
\text { les, bosques y tierras de conservación. Mayor } \\
\text { DAP por los servicios recreativos y estéticos. }\end{array}$ \\
\hline
\end{tabular}

Fuente: Elaboración propia.

Algunos críticos sostienen que la función de precios hedónicos no se puede estimar, debido al costo de la obtención de los bienes recreativos es exógena. Arguea y Hsiao (1993) muestran que si los atributos son independientes, los consumidores toman la decisión mejor 
representada por una función lineal de atributos de precio. El método de precios hedónicos se deriva de la misma premisa básica de la maximización de la utilidad a través de una elección de los sitios que difieren en los atributos y los costos. Contrariamente a las afirmaciones de Bockstael y Hanemann (1987), este método no puede descartarse simplemente porque la teoría es incorrecta o el enfoque para modelar la demanda de atributos es incompatible con la conducta.

\section{VALORACIÓN DE ACTIVOS MULTICRITERIO}

El MVAm ha sido diseñado para entornos en los que hay escasa información disponible (cuadro 5). Puede ser entendida como un método mixto que combina dos de las metodologías multicriterio más extendidas: PJA y PM. El PJA permite cuantificar las variables cualitativas e incorporar las intensidades en las preferencias, $y$ la PM captura la información proporcionada por la escasa información y la actitud del evaluador en el proceso de valoración. Aznar et ál., (2011) mencionan que la propuesta tiene una especial relevancia en situaciones de falta de información, mercados indeterminados y una baja tasa de transacciones, como suele ser el caso de la valoración agrícola.

CUADRO 5

CARACTERÍSTICAS DE LOS ESTUDIOS DE VALORACIÓN DE ACTIVOS MULTICRITERIO

\begin{tabular}{llllll}
\hline AUTOR & AÑO & LUGAR & ESTUDIO & MUESTRA & PRINCIPALES RESULTADOS \\
\hline $\begin{array}{l}\text { Aznar, J. } \\
\text { et ál. }\end{array}$ & 2011 & España & $\begin{array}{l}\text { Parcelas de } \\
\text { frutales }\end{array}$ & $\begin{array}{l}\text { Expertos } \\
\left(\begin{array}{l}1 \\
y\end{array}\right)\end{array}$ & $\begin{array}{l}\text { Obtuvieron el valor de la unidad de superficie de } \\
\text { cada activo valorado, en función de todos los datos } \\
\text { comparables utilizados y de su importancia o } \\
\text { ponderación. }\end{array}$ \\
\hline
\end{tabular}

Fuente: Elaboración propia.

De Salvo et ál. (2014) mencionan que el PJA presenta la ventaja de permitir una ordenación cuantitativa de los distintos tipos de paisajes $y$ discriminar entre los entrevistados cuyas respuestas son consistentes y aquellas que no lo son. Por tanto, en el caso en que no se necesite entrevistar a un menor número de personas (caso de las valoraciones realizadas con expertos), las ventajas que proporciona este método pueden llegar a compensar sus inconvenientes.

\section{COMBINACIÓN DE MÉTODOS}

En el resto de los documentos se aplica la combinación de dos o más métodos para estimar el valor monetario de los efectos generados por la agricultura en el ambiente, que incluyen tanto preferencias declaradas para determinar la disposición a pagar, como preferencias reveladas para estimar los gastos aproximados que están involucrados en la participación en actividades estudiadas. Estas diferentes técnicas de valoración rara vez se combinan (Willemen et ál., 2010), a pesar del reconocimiento de que la valoración monetaria es importante para el manejo eficaz de los ecosistemas.

En el cuadro 6, se mencionan las características de los documentos. En el primer documento se utilizaron los costos de reemplazo y la valoración contingente, mientras que en el segundo fue el análisis costo-efectividad, costos de transacción y experimentos de elección. Dachary-Bernard et ál. (2012) usaron los experimentos de elección y valoración contingente; mientras que en el documento siguiente utilizaron los experimentos de elección y los precios hedónicos. En el penúltimo estudio usaron el 
análisis conjunto y valoración contingente. Van Berkel et ál. (2014) usaron una combinación de experimentos de elección, valoración contingente, costo de viaje y precios hedónicos.

\section{CARACTERÍSTICAS DE LOS ESTUDIOS QUE PRESENTAN UNA COMBINACIÓN DE MÉTODOS}

\begin{tabular}{|c|c|c|c|c|c|}
\hline AUTOR & AÑO & LUGAR & ESTUDIO & MUESTRA & PRINCIPALES RESULTADOS \\
\hline $\begin{array}{l}\text { Almansa, C. } \\
\text { et ál. }\end{array}$ & 2012 & España & Erosión & 334 individuos & $\begin{array}{l}\text { La DAP varía con las variables } \\
\text { socioeconómicas y las variables } \\
\text { relacionadas con la actividad } \\
\text { agropecuaria. }\end{array}$ \\
\hline $\begin{array}{l}\text { Bakam, I. } \\
\text { et ál. }\end{array}$ & 2012 & Escocia & $\begin{array}{l}\text { Gases de efecto } \\
\text { invernadero }\end{array}$ & $\begin{array}{l}192 \text { granjas } \\
\text { agrícolas y } \\
\text { ganaderas }\end{array}$ & $\begin{array}{l}\text { La rentabilidad depende tanto de } \\
\text { la tasa de impuestos y la cantidad } \\
\text { de derechos de emisión gratuitos } \\
\text { asignados a los agricultores. }\end{array}$ \\
\hline $\begin{array}{l}\text { Dachary- } \\
\text { Bernard, J. } \\
\text { et ál. }\end{array}$ & 2012 & Francia & Paisajes agrícolas & $\begin{array}{l}284 \text { hogares y } \\
221 \text { individuos }\end{array}$ & $\begin{array}{l}\text { Altas preferencias por un componente } \\
\text { agrícola del paisaje, tierras de cultivo, } \\
\text { a favor de un programa de paisaje } \\
\text { extendido (paisaje agrícola y rural). }\end{array}$ \\
\hline $\begin{array}{l}\text { Duke, J. M. } \\
\text { et ál. }\end{array}$ & 2014 & $\begin{array}{l}\text { Estados } \\
\text { Unidos }\end{array}$ & $\begin{array}{l}\text { Preservación de } \\
\text { tierras agrícolas }\end{array}$ & 5315 parcelas & $\begin{array}{l}\text { La DAP se focaliza en conservar la } \\
\text { mayor cantidad de tierra, aunque } \\
\text { gran parte de la tierra tiene beneficios } \\
\text { netos bajos de preservación. }\end{array}$ \\
\hline $\begin{array}{l}\text { Gómez- } \\
\text { Limón, J. } \\
\text { et ál. }\end{array}$ & 2012 & España & $\begin{array}{l}\text { Implicaciones } \\
\text { ambientales en el } \\
\text { manejo del suelo }\end{array}$ & 758 individuos & $\begin{array}{l}\text { Mayor DAP por la adopción de las } \\
\text { prácticas agronómicas (cubierta } \\
\text { vegetal y picado e incorporación de los } \\
\text { restos de poda). }\end{array}$ \\
\hline $\begin{array}{l}\text { Sayadi, S. } \\
\text { et ál. }\end{array}$ & 2009 & $\begin{array}{l}\text { Medite- } \\
\text { rráneo }\end{array}$ & Paisaje agrícola & 163 encuestas & $\begin{array}{l}\text { Los atributos de la capa de vegetación, } \\
\text { el nivel de la construcción, y el nivel } \\
\text { de pendiente más importancia relativa } \\
\text { de las preferencias y DAP. El elemento } \\
\text { agrícola es lo más altamente estimado } \\
\text { estéticamente, y estimula fuertemente } \\
\text { la DAP. }\end{array}$ \\
\hline $\begin{array}{l}\text { Van Berkel, } \\
\text { D. et ál. }\end{array}$ & 2014 & $\begin{array}{l}\text { Países } \\
\text { Bajos }\end{array}$ & $\begin{array}{l}\text { Servicios de los } \\
\text { ecosistemas en } \\
\text { paisajes agrícolas }\end{array}$ & 115 encuestas & $\begin{array}{l}\text { Existe una demanda social por los } \\
\text { servicios culturales que tales paisajes } \\
\text { agrícolas proporcionan: edificios } \\
\text { culturales, líneas de árboles, lagos } \\
\text { y ríos, bosques y observación de la } \\
\text { fauna. }\end{array}$ \\
\hline
\end{tabular}

Fuente: Elaboración propia.

Baker y Ruting (2014) mencionan que los métodos de preferencias declaradas y reveladas suelen proporcionar diferentes medidas de bienestar; por ejemplo, el excedente del consumidor en el caso de los modelos de costo de viaje (DAP menor del precio pagado), precios implícitos en los precios hedónicos y modelos de elección (DAP por una unidad adicional de un atributo), y la DAP total en la valoración contingente.

Las estimaciones también pueden diferir cuando los métodos de preferencias declaradas incluyen algunos valores de no uso, que en general no son recogidos en las estimaciones de preferencias reveladas. Estas complicaciones 
sustentan una corriente en la literatura que combina los datos de ambas fuentes de preferencias declaradas y reveladas, sobre la base de que ninguno es perfecto, pero cada uno puede proporcionar una perspectiva diferente sobre el comportamiento (Adamowicz et ál. 1994).

\section{RECOMENDACIONES PARA LA VALORACIÓN ECONÓMICA DEL AMBIENTE}

Con el fin de ofrecer recomendaciones para el uso de los métodos de valoración económica y algunas consideraciones relacionadas con la regulación de las actividades agrícolas que contaminan al ambiente, en el diseño de políticas, se analizó el efecto causal sobre las características de la población; para aumentar la capacidad predictiva de los resultados cuando la misma intervención se introduce a una nueva área con características diferentes; es decir, si los resultados pueden ser replicados.

Los resultados de la aplicación de los métodos de preferencias declaradas pueden replicarse con éxito cuando se encuesta a una muestra diferente de personas, sin diferencias significativas entre las muestras y/o alta correlación con el tiempo (McConnell et ál. 1998).

Así, dentro de los documentos analizados, algunos estudios reportan el efecto de la intervención del promedio en la población, mientras que otros analizan cómo el impacto varía dependiendo de las condiciones socioeconómicas de los hogares. Ma et ál. (2011) mencionan que los programas de conservación del medio ambiente a menudo subsidian propietarios de tierras agrícolas para preservar los recursos y paisajes naturales, como bosques, humedales y pastizales, que apoyan los servicios de los ecosistemas públicos. Por lo tanto, políticas públicas rentables sobre los bienes públicos, como el secuestro de carbono o hábitat de vida silvestre deben adaptarse a la DAP de los propietarios para proporcionar estos servicios.

Los sectores más jóvenes y de mayores ingresos en la población, que a menudo se asocian con un mayor nivel de educación y por lo tanto, de acceso a la información, aumenta la DAP. Así, las campañas públicas que proporcionen información sobre la calidad del medio ambiente son una herramienta importante $y$ eficiente (Almansa et ál. 2012). Por lo tanto, para Jianjun et ál. (2013a) debe haber un aumento de las inversiones en los programas de educación y de comunicación pública que puedan reforzar estos conocimientos, con el fin de mejorar las actitudes $y$ las percepciones de las personas acerca de la protección de los ecosistemas agrícolas.

Además de lo anterior, se deben considerar los aspectos que son percibidos por las personas encuestadas como menos importantes o por los cuales no están dispuestos a pagar; estos pueden ser de utilidad en la detección de posibles conflictos asociados a las nuevas prácticas de manejo y planificación del sistema ambiental, ayudando a la comprensión de su efecto sobre el desempeño de las políticas de mitigación. Bakam et ál. (2012) sugieren incluir las actitudes de las personas entrevistadas a las nuevas políticas, tales como la resistencia al cambio, los comportamientos de preferencias por riesgo y los distintos motivos que maximicen la ganancia.

Aunque los métodos propuestos se ilustran para diversos estudios de caso, una política agrícola dirigida esperaría beneficios sociales más altos en términos de la conservación del paisaje, apoyo a la agricultura y desarrollo local, debido a que el análisis costo-beneficio contribuye a la formulación de mecanismos de política adecuados, lo que aumenta la conciencia pública y política de la importancia del problema, así como, ayuda a establecer prioridades de conservación. Tal como mencionan Tait et ál. (2011), una estrategia de manejo colaborativa que abarque diversos intereses es crucial para la formación de políticas que sean aceptables para el público en general. La inclusión de los valores económicos es un componente importante porque revela el nivel y la importancia relativa de las preferencias del público, dando como resultado políticas que se puedan utilizar para asignar el manejo de los recursos eficientemente.

Asimismo, Mayrand et ál. (2003) mencionan que la reforma del sistema de apoyo agrícola hacia una nueva generación de políticas agrícolas se puede delinear mediante una serie de principios que mejoran la coherencia, 
eficiencia y efectividad de las diversas políticas. Dentro de la integración y coherencia, los subsidios y otras medidas de apoyo a la agricultura del sistema mexicano operan dentro de un entorno político complejo, donde las medidas de apoyo y la reglamentación ambiental van en dirección opuesta, trayendo como consecuencia la reducción de la efectividad y eficiencia de la estructura de protección ambiental.

\section{CONCLUSIONES}

La valoración contingente es una de las metodologías más utilizadas para asignar valor monetario a los recursos ambientales $y$ aunque presenta ciertas limitaciones, es un método prometedor para valorar de forma directa el ecosistema y podría ser utilizado para obtener información útil para la toma de decisiones de política pública. Permite conocer los procesos cognitivos, la racionalidad y la naturaleza de las preferencias de los consumidores respecto de determinado bien ambiental, sean públicos o privados, $y$ así obtener un valor económico total, derivado de los valores de uso, opción y existencia.

La disposición a pagar varía en los beneficiarios con diferentes características socioeconómicas, lo cual se ve influenciado positivamente por los sectores más jóvenes, la población con mayores ingresos. Estos factores están muy asociados con un mayor nivel de educación y por lo tanto, de acceso a la información, lo que mejora las actitudes y las percepciones de las personas acerca de la protección de los ecosistemas agrícolas.

Si bien, existen diversos métodos de valoración, a veces no tienen aplicabilidad en el sector agrícola, pues no se han elaborado pensando en la facilidad de hacer las mediciones $y$ en la frecuencia con la que se recoge la información, asimismo, no se han establecido límites estandarizados para los sistemas y no se ha analizado cómo hacer que estas metodologías ayuden a mejorarlos o sirvan para dar subsidios por beneficios ambientales, tales como, el pago por servicios de los ecosistemas y las medidas de adaptación.

Una cuestión importante de los resultados finales de la revisión de los métodos de valoración reveló que los daños son generalmente reconocidos como críticos, sentando las bases para la implementación de políticas públicas enfocadas en mitigar los problemas estudiados. Sin embargo, el estudio también mostró que muchas simplificaciones se han hecho en funciones de daño existente. Una razón probable destacada por el análisis es que los datos existentes sobre los daños de la agricultura son escasos. En consecuencia, mientras que la mayoría de los esfuerzos de investigación se han centrado en daños relacionados con la recreación y el turismo, dejando de lado los problemas ocasionados por los cultivos.

El desarrollo de los métodos disponibles para la valoración y sus aplicaciones han sido realizados principalmente en países desarrollados, los cuales generalmente utilizan una mayor cantidad de subsidios, en contraste con los países en desarrollo. Por lo tanto, se esperaría que para aplicar estudios de valoración ambiental en todos los países, es necesario que no prevalezca esta diferencia en la aplicación de los subsidios.

La valoración económica de los problemas ambientales ocasionados por las actividades agrícolas, no es tarea fácil. Implica llevar a cabo cambios orientados a fomentar una agricultura más sostenible. Debido a que tanto los beneficios de esta actividad, como los problemas ambientales $y$ sociales, no se observan de forma tan rápida como los económicos. Los agricultores se enfocan en la producción, sin considerar la cantidad de insumos utilizados y como consecuencia, no se consideran los daños ambientales. Por ello hay que concientizar a los políticos, administradores, técnicos y agricultores, sobre la importancia de considerar todo conjuntamente; ver al ambiente como un sistema integrador y no solo como un receptor de los residuos de producción.

\section{BIBLIOGRAFÍA}

LIBROS

Diamond, Peter A. y Jerry A. Hausman. 1993. "On contingent valuation measurement of non-use values". En Contingent valuation: a critical assessment, editado 
por Hausman J. A., 3-38. Amsterdam: North Holland.

Freeman III, A. Myrick. 2003. The Measurement of Environment and Resource Values: Theory and Method. 2nd ed. Resources for the Future, 512. Washington, DC. USA.

Riera, Pere. 1994. Manual de valoración contingente. Instituto de Estudios Fiscales.

Torgerson, Carole. 2003. Systematic reviews. Continuum. Gran Bretaña: Cornwall.

PUBLICACIONES PERIÓDICAS

Adamowicz, Wiktor; Jordan Louviere y Michael Williams. 1994. "Combining revealed and stated preference methods for valuing environmental amenities". Journal of Environmental Economics and Management 26: 271-292.

Afroz, Rafia; Keisuke Hanaki y Kiyo HasegawaKurisu. 2009. "Willingness to pay for waste management improvement in Dhaka city, Bangladesh". Journal of Environmental Management 90: 492503. DOI: 10.1016/j.jenvman.2007.12.012.

Almansa, Carmen; Javier Calatrava, y José Miguel Martínez-Paz. 2012. "Extending the framework of the economic evaluation of erosion control actions in Mediterranean basins". Land Use Policy 29, n. ${ }^{\circ}$ 2: 294-308. DoI: 10.1016/j. landusepol.2011.06.013.

Alpízar, Francisco; Fredrik Carlsson y Peter Martinsson. 2003. "Using Choice Experiments for Non-Market Valuation". Economic Issues 8: 83-110.

Arguea, Nestor M. y Cheng Hsiao. 1993. "Econometric Issues of Estimating Hedonic Price Functions: With an Application to thr us Market for Automobiles". Journal of Econometrics 56, n. ${ }^{\circ} 1-2: 243-267$.

Aznar, Jerónimo; Francisco Guijarro y José María Moreno-Jiménez. 2011. "Mixed valuation methods: a combined AHPGP procedure for individual and group multicriteria agricultural valuation”. Annals of Operations Research 190, n. ${ }^{\circ}$ 1: 221-238. DOI: $10.1007 / \mathrm{s} 10479-009$ 0527-2.

Bakam, Innocent; Bedru Babulo Balana $y$ Robin Matthews. 2012. "Costeffectiveness analysis of policy instruments for greenhouse gas emission mitigation in the agricultural sector". Journal of Environmental Management 112: 33-44. DOI: 10.1016/j. jenvman.2012.07.001.

Baker, Rick y Brad Ruting. 2014. "Environmental Policy Analysis: A Guide to Non-Market Valuation". Productivity Commission Staff Working Paper: 151. Camberra, Australia.

Barrena, José; Laura Nahuelhual; Andrea Báez; Ignacio Schiappacasse y Claudia Cerda. 2014. "Valuing cultural ecosystem services: Agricultural heritage in Chiloé island, southern Chile". Ecosystem Services 7: 66-75. DOI: 10.1016/j. ecoser.2013.12.005.

Barton, David N. y Avinandan Taron. 2010. "Valuing irrigation water using surveybased methods in the Tungabhadra River Basin, India”. Irrigation and Drainage Systems 24: 265-277. DOI: 10.1007/ s10795-010-9098-2.

Bockstael, Nancy Elaine y W. Michael Hanemann. 1987. "Modelling recreational demand in a multiple site framework". Water Resources Research 23, n. 5: 951-960.

Carlsson, Fredrik y Martinsson, Peter. 2001. "Do Hypothetical and Actual Marginal Willingness to Pay Differ in Choice Experiments? Application to the Valuation of the Environment". Journal of Environmental Economics and Management 41: 179-92.

Carson, Richard T. y Theodore Groves. 2007. "Incentive and informational properties of preference questions". Environmental and Resource Economics 37:181-210.

Castro, Antonio J.; Berta Martín-López; Marina García-Llorente; Pedro A. Aguilera; Enrique López y Javier Cabello. 2011. "Social preferences regarding the delivery of ecosystem services in a 
semiarid Mediterranean region". Journal of Arid Environments 75, n. ${ }^{\circ}$ 11: 12011208. DOI: 10.1016/j.jaridenv.2011.05.013

Dachary-Bernard; Jeanne y Tina Rambonilaza. 2012. "Choice experiment, multiple programmes contingent valuation and landscape preferences: How can we support the land use decision making process?". Land Use Policy 29, n..$^{\circ}$ 4: 846-854. DOI: $10.1016 / \mathrm{j}$. landusepol.2012.01.002.

De Salvo, María Cristina; María Vallés-Planells; Vicente Estruch Guitart y Bruno Francesco Nicolò. 2014. "Valoración estética del paisaje mediante los modelos AHP y percepción visual. Aplicación al paisaje de olivar de la La Piana di Gioia Tauro ". Revista Española de Estudios Agrosociales y Pesqueros 239: 87-106.

Diamond, Peter A. y Jerry A. Hausman. 1994. "Contingent Valuation: Is Some Number Better than No Number?". Journal of Economic Perspectives 8, n. ${ }^{\circ}$ 4: 45-64.

Duke, Joshua M.; Steven J. Dundas; Robert J. Johnston y Kent D. Messer. 2014. "Prioritizing payment for environmental services: Using nonmarket benefits and costs for optimal selection". Ecological Economics 105: 319-329. DOI: 10.1016/j. ecolecon.2014.06.014.

Eyckmans, Johan; Simon De Jaeger y Sandra Roussea. 2013. "Hedonic Valuation of Odor Nuisance Using Field Measurements: a Case Study of an Animal Waste Processing Facility in Flanders". Land Economics 89, n. ${ }^{\circ}$ 1: 53-75.

Flores Crespo, Pedro. 2013. "El enfoque de la política basado en la evidencia: Análisis de su utilidad para la educación de México". Revista Mexicana de Investigación Educativa 18, n. ${ }^{\circ}$ 56:265-290.

Gómez-Limón, José A. y Jesús Barreiro-Hurlé. 2012. "Valoración económica de las técnicas sostenibles de manejo del suelo en el olivar andaluz". Cuadernos de economía 35: 158-171.

Grammatikopoulou, Ioanna; Eija Pouta and Maija Salmiovirta. 2013. "A locally designed payment scheme for agricultural landscape services". Land Use Policy. 32: 175-185. DoI: 10.1016/j. landusepol.2012.10.010.

Hanemann, W. Michael. 1994. "Valuing the Environment Through Contingent Valuation". Journal of Economic Perspectives 8, n. ${ }^{\circ}$ 4: 19-43.

Haro Martínez; Alma Angelina y Cristina Taddei Bringas. 2010. "Valoración ambiental: aportaciones, alcances y limitaciones. Problemas del Desarrollo". Revista Latinoamericana de Economía 41, n. ${ }^{\circ}$ 160: 209-221.

Hausman, Jerry. 2012. "Contingent Valuation: From Dubious to Hopeless". Journal of Economic Perspectives 26, n. ${ }^{\circ}$ 4: 43-56. DOI:10.1257/jep.26.4.43.

Jianjun, Jin; Jiang Chong y Li Lun. 2013a. "The economic valuation of cultivated land protection: A contingent valuation study in Wenling City, China". Landscape and Urban Planning 119: 158-164. DOI: 10.1016/j.landurbplan.2013.06.010.

Jianjun, Jin; Jiang Chong; Truong Dang Thuy y Li Lun. 2013b. "Public preferences for cultivated land protection in Wenling City, China: a choice experiment study". Land Use Policy 30, n. 1: 337-343. Dor: 10.1016/j.landusepol.2012.04.003.

Kahneman, Daniel y Jack Knetsch. 1992. "Valuing public goods: The purchase of moral satisfaction". Journal of Environmental Economics and Management 22: 57-70.

Kealy, Mary Jo; Mark Montgomery y John F. Dovidio. 1990. "Reliability and predictive validity of contingent values: does the nature of the good matter?". Journal of Environmental Economics and Management 19: 244-263.

Lancaster, Kelvin J. 1966. "A new approach to consumer theory". Journal of Political Economy 74, 132-157.

Louviere, Jordan J. 1988. "Conjoint Analysis Modelling of Stated Preferences. A Review of Theory, Methods, Recent Developments and External Validity". Journal of Transport Economics and Policy 22: 93-119. 
Ma, Shan y Scott M. Swinton. 2011. "Valuation of ecosystem services from rural landscapes using agricultural land prices". Ecological Economics 70, n. ${ }^{\circ}$ 9: 1649-1659. DOI: $10.1016 / \mathrm{j}$. ecolecon.2011.04.004.

Mayrand, Karel; Stéphanie Dionne; Marc Paquin e Isaak Pageot-LeBel. 2003. "The Economic and Environmental Impacts of Agricultural Subsidies: An Assessment of the 2002 US Farm Bill \& Doha Round, Montreal". Unisféra International Centre. McConnell, Kenneth E.; Ivar E. Strand y Sebastián Valdés. 1998. "Testing Temporal Reliability and Carry-over Effect: The Role of Correlated Responses in Test-retest Reliability Studies". Environmental and Resource Economics 12: 357-374.

Mesa-Jurado, M. Azahara; Julia MartinOrtega; Eric Ruto y Julio Berbel. 2012. "The economic value of guaranteed water supply for irrigation under scarcity conditions". Agricultural Water Management 113: 10-18. DOI: 10.1016/j. agwat.2012.06.009.

Mezgebo, Alem; Worku Tessema y Zebene Asfaw. 2013. "Economic Values of Irrigation Water in Wondo Genet District, Ethiopia: An Application of Contingent Valuation method". Journal of Economics and Sustainable Development 4, n. ${ }^{\circ}$ 2: 23-37.

Moon, Wanki y Jacob Wayne Griffith. 2011. "Assessing holistic economic value for multifunctional agriculture in the US". Food Policy 36, n. ${ }^{\circ}$ 4: 455-465. DoI: 10.1016/j.foodpol.2011.05.003.

Ojeda, Monica Ilija; Alex S. Mayer y Barry D. Solomon. 2008. "Economic valuation of environmental services sustained by water flows in the Yaqui River Delta". Ecological Economics 65, n. 1:155-166.

Parra, Salvador; Fernando J. Aguilar y Javier Calatrava. 2008. "Decision modelling for environmental protection: The contingent valuation method applied to greenhouse waste management". Biosystems Engineering 99, n. ${ }^{\circ}$ 4: 469-477.
Pérez Vázquez, Arturo y Cesáreo Landeros Sánchez. 2009. "Agricultura y deterioro ambiental". Elementos: Ciencia y cultura 16, n. ${ }^{\circ} 73:$ 19-25.

Poudel, Diwaker y Fred H. Johnsen. 2009. "Valuation of crop genetic resources in Kaski, Nepal: farmers' willingness to pay for rice landraces conservation". Journal of Environmental Management 90, n. ${ }^{\circ}$ : 483-491.

Rosen, Sherwin. 1974. "Hedonic Prices and Implicit Markets: Product Differentiation in Pure Competition". Journal of Political Economy 82: 34-55.

Ryan, Robert L. 2011. "The social landscape of planning: integrating social and perceptual research with spatial planning information". Landscape Urban Plan 100: 361-363.

Sanz, Jordi. 2013. "La aportación de las revisiones sistemáticas al diseño de las políticas públicas: de la anécdota a la evidencia”. Avaluació per al Bon Govern 7.

Sayadi, Samir, M.; Carmen González-Roa y Javier Calatrava-Requena. 2009. "Public preferences for landscape features: The case of agricultural landscape in mountainous Mediterranean areas". Land Use Policy 26, n. ${ }^{\circ}$ 2: 334-344.

Smith, V. Kerry. 1993. "Nonmarket valuation of environmental resources: an interpretative appraisal". Land Economics 69: 1-26.

Smith, V. Kerry y Laura L. Osborne. 1996. "Do contingent valuation estimates pass a 'scope' test? A meta-analysis". Journal of Environmental Economics and Management 31: 287-301.

Tait, Peter; Ramesh Baskaran; Ross Cullen y Kathryn Bicknell. 2011. "Valuation of agricultural impacts on rivers and streams using choice modelling: A New Zealand case study". New Zealand Journal of Agricultural Research 54, n. ${ }^{\circ}$ 3: 143-154.

Van Berkel, Derek B. y Peter H. Verburg. 2014. "Spatial quantification and valuation of cultural ecosystem services in an agricultural landscape". Ecological 
Indicators 37: 163-174. DOI: 10.1016/j. ecolind.2012.06.025.

Venkatachalam, Lingappan. 2004. "The contingent valuation method: a review". Environmental Impact Assessment Review 24, n. ${ }^{\circ}$ 1: 89-124.

Willemen, Louise, Lars Hein y Peter $\mathrm{H}$. Verburg. 2010. "Evaluating the impact of regional development policies on future landscape services". Ecological Economics 69: 2244-2254.

OTROS

National Oceanic and Atmospheric Administration-NOAA. 1993. Report of the NOAA Panel on contingent valuation.
National Oceanic and Atmospheric Administration. Federal Register. 58: 4602-4614.

Kristrom, Bengt. 1995. Theory and Applications ot the Contingent Valuation Method. Simposio Economía ambiental: Valoración, recursos naturales y politica económica. Barcelona. Universidad Internacional Menéndez y Pelayo.

World Bank. 1992. World Bank Development Report. Oxford Univ. Press. New York. DOI: 10.1596/978-0-19-520876-4

Fecha de ingreso: 26/09/2015 Fecha de aprobación:12/02/2016 
